\title{
Exact solution to a class of functional difference equations with application to a moving contact line flow
}

\author{
J. B. LAWRIE ${ }^{1}$ and A. C. KING \\ 'Department of Mathematics and Statistics, Brunel University, Uxbridge, Middlesex UB8 3PH, UK \\ ${ }^{2}$ Department of Mathematics, Keele University, Keele, Staffs ST5 5BG, UK
}

(Received in revised form 21 December 1992)

\begin{abstract}
A new integral representation for the Barnes double gamma function is derived. This is canonical in the sense that solutions to a class of functional difference equations of first order with trigonometrical coefficients can be expressed in terms of the Barnes function. The integral representation given here makes these solutions very simple to compute. Several well-known difference equations are solved by this method, and a treatment of the linear theory for moving contact line flow in an inviscid fluid wedge is given.
\end{abstract}

\section{Introduction}

There are many physical problems that can be modelled using a steady state field equation. For example, Laplace's equation describes ideal fluid flows and Helmholtz' the propagation of acoustic waves. It is the geometrical configuration and the nature of the boundary conditions, not the physical origin or governing equation, that determines the mathematical complexity and tractability of a given problem. The boundary conditions may be of mixed form, be discontinuous or have a smoothly varying parameter. The appropriate method of solution will, again, depend on the geometry and type of boundary conditions involved. A vast range of mathematical techniques are available, many of which (including the notable Wiener-Hopf technique (Noble, 1968)) rely on the use of integral transforms.

The Wiener-Hopf technique is applicable to two-part problems in which an infinite boundary condition is discontinuous, having one form for $z<0$, say, and another for $z>0$. The method hinges on finding a product factorization for the Wiener-Hopf kernelthat is, in finding a representation for the kernel as the product of two functions which are analytic in overlapping upper and lower halves of the complex plane respectively. The Wiener-Hopf kernel is fundamental to the solution of a given problem containing information about its physical features. For example, it may define the wavenumbers of any travelling waves or specify the form of the radiated field.

However, only a small class of boundary value problems can be solved by means of the Wiener-Hopf technique and the application of integral transforms to other types may focus mathematical attention in a direction that is, in some ways, equivalent to finding a factorization for a Wiener-Hopf kernel. For example, there is a diverse range of boundary value problems that, under integral transform, give rise to a functional difference equation (fde) which, like a Wiener-Hopf kernel, is canonical to the physical solution in that it 
contains vital information about its properties. Typically such boundary value problems may have a wedge geometry with mixed boundary conditions (for example, Isaccson, 1950; Lauwerier, 1961; Faulkner, 1964; Packham, 1989). Alternatively, they may have a boundary condition that is applied over an infinite range, $-\infty<z<\infty$ say, and which contains a parameter that varies smoothly with $z$ (Roseau, 1976; Evans, 1984). To obtain an analytic solution to the boundary value problem, a closed form solution to the fde must first be found.

There are many classes of fde and by no means all can be solved analytically. We consider only linear fdes and for a comprehensive discussion of the analytic solution of linear difference equations with rational coefficients the reader is referred to Milne-Thompson (1933). Briefly, the best-known methods include the use of the gamma function, Alexeiewsky's $G$ function and, in particular, integral transforms. Koiter (1955) demonstrates the use these techniques whilst obtaining the solution to the fde

$$
T_{0}(s+1)=-2 s \cot (\pi s) T_{0}(s) .
$$

The Maliuzhnets' function, defined by the fde

$$
M_{\alpha}(v+2 \alpha)-\cot \left\{\frac{1}{2}\left(v+\frac{1}{2} \pi\right)\right\} M_{\alpha}(v-2 \alpha)=0
$$

together with the conditions $M_{\alpha}(0)=1$ and $M_{\alpha}(v)=O(\exp \{|\pi \mathscr{T}(v) / 8 \alpha|\})$ as $\mathscr{T}(v) \rightarrow \infty$ (see Maliuzhnets, 1958), may be expressed in integral form (see Appendix): its properties and use in solving a certain class of fde has been discussed by a variety of authors (for example, Tuzhilin, 1971; Lipsyzc, 1975, 1980; Hongo, 1986). Williams (1959), when considering electromagnetic diffraction problems, presents the solution to a fde of similar type first in terms of the double gamma function as defined by Barnes (1899), and then as a finite product of elementary trigonometric functions (the source of the latter being unclear). Lawrie (1990), considering an fde of the same class, goes further in using the recurrence relations for the Barnes double gamma function (see Appendix) to connect the two forms of solution.

In this article we consider first-order fdes of the class:

$$
A(\alpha, s) f(s)-B(\alpha, s) f(s+1)=0,
$$

into which category those of Maliuzhnets (1958), Van Dantzig (1958), Williams (1959) and Lawrie (1990) fall. Here $\alpha$ is a real parameter and $A(\alpha, s), B(\alpha, s)$ denote trigonometrical functions of the form $\sin \alpha(s+\beta)$ or a product of such functions (where $\beta$ is real). Functional difference equations of this type are particularly common, arising in the solution of boundary value problems from many areas of applied mathematics. In the case of linear water waves, the typical boundary value problem consists of Laplace's equation in a wedge $(0 \leqslant \theta \leqslant \alpha)$ with different boundary conditions on the rays $\theta=0$ and $\theta=\alpha$ (see, for example, Ehrenmark, 1987). One boundary condition is often either Neumann or Dirichlet in form: $\partial \phi / \partial \theta$ or $\phi$ given where $\phi$ is the fluid velocity potential. However, the other boundary condition may be a linear combination of terms in $\phi, \partial \phi / \partial r$ and $\partial \phi / \partial \theta$. On applying the Mellin transform to such a boundary value problem, it is found that the derivatives with respect to $r$ give rise to a fde whilst the derivatives with respect to $\theta$ cause the quantities $A(\alpha, s), B(\alpha, s)$ to be trigonometrical in form.

If $\alpha=\pi$, so that the wedge becomes a half plane, there are a variety of well-established techniques for solving the two-part boundary value problem. These include integral 
equation techniques, conformal mapping and, of course, the Wiener-Hopf technique. A survey of half-plane problems has recently been given by Varley \& Walker (1989), who also present some new methods of solution. However, it is the intention of this work to consider only genuine wedge problems $(\alpha<\pi)$ where the neither classical nor Varley \& Walker's methods seem to be readily applicable.

The class of fde described by (1.3) is well documented, and the thrust of this paper is in presenting a simple analytic algorithm which enables the solution to any fde of this form to be written down by inspection. Fundamental to the method is the derivation of an integral representation for the logarithm of the Barnes unsymmetric double gamma function. This expression is derived in $\S 2$ and has two distinct advantages. Firstly, products and quotients of the Barnes double gamma function are easily combined into one integral expression and, secondly, the integral has a finite range of integration and is easy to compute. In $\S 3$ it is shown that the solution of fdes of the form of (1.3) can always be formulated in terms of quotients of the Barnes double gamma function. In $\S 4$ it is demonstrated that an easily computable analytic expression for the solution of such an fde can thus be written down by inspection. It is also shown that, for the special case $\alpha=p \pi / 2 q$, the general solution can be simplified to a finite product of trigonometrical functions without any need for an integral expression. In $\S 5$ a treatment of a moving contact line flow in an inviscid fluid wedge is given. The displacement of the contact point, which is driven along a solid surface by surface tension, is expressed and computed using the Barnes double gamma function. The article is concluded with a brief discussion in $\S 6$. Finally, the infinite product representation for the Barnes double gamma function is given in the Appendix. This is then used as an alternative route to the integral representation, confirming the analysis of $\$ 2$. The duplication formula is derived, the Barnes double gamma function is plotted for various values of the parameter $\delta$ and its connection with the elementary trigonometric functions, Alexeiewsky's $G$ function and Maliuzhnets' function is clarified.

\section{An integral expression for the Barnes double gamma function}

In this section a simple integral representation for the Barnes double gamma function is derived by means of expressing its logarithmic derivative as a Mellin transform. The Barnes double gamma function is defined by the difference equation

$$
G(s+1, \delta)=\Gamma(s / \delta) G(s, \delta)
$$

with normalization condition

$$
G(1, \delta)=1 .
$$

On taking the logarithm of each side of (2.1) and differentiating twice with respect to $s$ it is found that,

$$
f(s+1)-f(s)=\frac{1}{\delta^{2}} \psi^{\prime}(s / \delta)
$$

where the prime denotes differentiation with respect to the argument and

$$
\begin{aligned}
& \psi(z)=\frac{\mathrm{d}}{\mathrm{d} z}\{\ln \Gamma(z)\} \\
& f(s)=\frac{\mathrm{d}}{\mathrm{d} s}\left\{\frac{G^{\prime}(s, \delta)}{G(s, \delta)}\right\}
\end{aligned}
$$


An exact solution to the first order fde (2.3) may be obtained as follows. Let

$$
f(s)=\int_{0}^{\infty} \frac{F(r)\left\{r^{s-1}-j(r)\right\}}{(r-1)} \mathrm{d} r,
$$

where $F(r)$ and $j(r)$ are unknown functions. It is worth noting that the literature contains many examples whereby an fde has been solved through representing the required function as an integral. The form of integral chosen varies, for example, Peters (1952) utilizes the Cauchy integral, Koiter (1955) and Roseau (1958) the inverse Laplace transform, and Evans (1984) the Fourier integral. However, the representation (2.4) is non-standard in that it contains two unknown functions. The function $j(r)$ is introduced to ensure convergence in the final integral representation for $\ln G(s, \delta)$; it is not sufficient to assume that $j(r)=1$ as in the case of Evans (1984). Substituting this expression into the left hand side of (2.3), it is found that

$$
\int_{0}^{\infty} F(r) r^{s-1} \mathrm{~d} r=\frac{1}{\delta^{2}} \psi^{\prime}(s / \delta) .
$$

This is a standard Mellin transform, and it follows that

$$
F(r)=H(1-r) \frac{\ln r}{r^{\delta}-1},
$$

where $H(\cdot)$ is the Heaviside function. Thus, from (2.4), it is seen that

$$
f(s)=\int_{0}^{1} \frac{\ln r}{(r-1)\left(r^{\delta}-1\right)}\left\{r^{s-1}-j(r)\right\} \mathrm{d} r .
$$

Integrating (2.6) twice with respect to $s$ it is found that

$$
\ln G(s, \delta)=\int_{0}^{1} \frac{\ln r}{(r-1)\left(r^{\delta}-1\right)}\left\{\frac{r^{8-1}}{(\ln r)^{2}}-\frac{s^{2}}{2} j(r)-s k(r)\right\}-l(r) \mathrm{d} r
$$

where $j(r), k(r), l(r)$ are as yet undetermined. The functions $j(r)$ and $k(r)$ are chosen so that this expression satisfies relation (2.1), i.e.

$$
\int_{0}^{1} \frac{1}{\left(r^{\delta}-1\right)}\left\{\frac{r^{s-1}}{\ln r}-s \frac{\ln r}{(r-1)} j(r)-\frac{\ln r}{(r-1)}\left[k(r)+\frac{1}{2} j(r)\right]\right\} \mathrm{d} r=\ln \Gamma(s / \delta) .
$$

Abramowitz \& Stegun (1972) give a standard integral representation for the quantity ln $\Gamma(z)$. It is convenient to write this in the form

$$
\ln \Gamma(s / \delta)=\int_{0}^{1} \frac{1}{\left(r^{\delta}-1\right)}\left\{\frac{r^{s-1}}{\ln r}-s \frac{r^{\delta-1}\left(r^{\delta}-1\right)}{\delta \ln r}+\frac{r^{\delta-1}\left(r^{\delta}-2\right)}{\ln r}\right\} \mathrm{d} r .
$$

Comparison of (2.9) and (2.11) now yields

and

$$
\begin{gathered}
j(r)=\frac{r^{\delta-1}(r-1)\left(r^{\delta}-1\right)}{\delta(\ln r)^{2}} \\
k(r)=\frac{r^{\delta-1}(r-1)\left(r^{\delta}-1\right)}{(\ln r)^{2}}\left\{\frac{2-r^{\delta}}{r^{\delta}-1}-\frac{1}{2 \delta}\right\} .
\end{gathered}
$$

Thus, it remains only to determine $l(r)$. This can be done by considering the singularity structure of the integrand of (2.8) close to the point $r=1$. The integrand is expanded to 
third order in powers of $(r-1)$ and $l(r)$ is determined by eliminating singularities in the terms that are independent of $s$. It is then found that

$$
l(r)=\frac{1}{\delta(r-1)^{3}}-\frac{1}{2(r-1)^{2}}+\frac{\delta^{2}+3 \delta+1}{12 \delta(r-1)}
$$

The resulting integral representation is that for $\{\ln G(s, \delta)+\ln C\}$ where $C$ is a constant and is determined using the normalization condition (2.2). Thus, finally, it is found that

$$
\begin{aligned}
G(s, \delta)=\exp \left\{\int _ { 0 } ^ { 1 } \left\{\frac{r^{s-1}}{(r-1)\left(r^{\delta}-1\right)}-\frac{s^{2}}{2 \delta} r^{\delta-1}-s r^{\delta-1}\right.\right. & \left\{\frac{2-r^{\delta}}{r^{\delta}-1}-\frac{1}{2 \delta}\right\}-r^{\delta-1} \\
& \left.\left.+\frac{1}{(r-1)}-\frac{r^{\delta-1}}{(r-1)\left(r^{\delta}-1\right)}\right\} \frac{\mathrm{d} r}{\ln r}\right\} .
\end{aligned}
$$

This integral representation is convergent for all $s$ such that $\operatorname{Re}(s)>0$ and for all $\delta>0$. Furthermore, it has the advantage of being easily computable for all real values of $s$ and $\delta$ and, thus, clearly increases the utility of the Barnes double gamma function. A graph showing the variation of $\ln \{G(s, \delta)\}$ for various values of $\delta$ is shown in Fig. 3 of the Appendix.

\section{Solution of a class of functional difference equations}

It is now shown that the solution to a certain class of fde may always be expressed in terms of quotients of the Barnes double gamma function. The class of fde to be considered is

$$
A(\alpha, s) f(s)-B(\alpha, s) f(s+1)=0 .
$$

Here quantities $A(\alpha, s), B(\alpha, s)$ have the form

$$
A(\alpha, s)=\prod_{i=1}^{l} \sin \left\{\alpha\left(s+\beta_{i}\right)\right\} \prod_{j=1}^{J} \cos \left\{\alpha\left(s+\tau_{j}\right)\right\}
$$

and

$$
B(\alpha, s)=\prod_{k=1}^{k} \sin \left\{\alpha\left(s+\mu_{k}\right)\right\} \prod_{l=1}^{L} \cos \left\{\alpha\left(s+\nu_{l}\right)\right\}
$$

where $\alpha, \beta_{i}, \tau_{j}, \mu_{k}, \nu_{l}$ are real. Thus, using the gamma function representation for sine and cosine, (3.1) may be rewritten as

$$
\frac{f(s)}{f(s+1)}=\pi^{\gamma} \prod_{i, j, k, l} \frac{\Gamma\left\{\alpha\left(s+\beta_{i}\right) / \pi\right\} \Gamma\left\{1-\alpha\left(s+\beta_{i}\right) / \pi\right\} \Gamma\left\{\frac{1}{2}+\alpha\left(s+\tau_{j}\right) / \pi\right\} \Gamma\left\{\frac{1}{2}-\alpha\left(s+\tau_{j}\right) / \pi\right\}}{\left.\Gamma\left(s+\mu_{k}\right) / \pi\right\} \Gamma\left\{1-\alpha\left(s+\mu_{k}\right) / \pi\right\} \Gamma\left\{\frac{1}{2}+\alpha\left(s+\nu_{l}\right) / \pi\right\} \Gamma\left\{\frac{1}{2}-\alpha\left(s+\nu_{l}\right) / \pi\right\}}
$$

where $\gamma=K+L-I-J$. Defining $\delta=\pi / \alpha$ and rewriting each gama function in terms of the Barnes double gamma function (see Appendix), is found that

$$
\begin{aligned}
\frac{f(s)}{f(s+1)}= & \pi^{\gamma} \prod_{i . j . k . l}\left\{\frac{G\left(1+s+\beta_{i}, \delta\right) G\left(1+\delta-s-\beta_{i}, \delta\right)}{G\left(s+\beta_{i}, \delta\right) G\left(\delta-s-\beta_{i}, \delta\right)}\right. \\
& \times \frac{G\left(1+\frac{1}{2} \delta+s+\tau_{j}, \delta\right) G\left(1+\frac{1}{2} \delta-s-\tau_{j}, \delta\right) G\left(s+\mu_{k}, \delta\right)}{G\left(\frac{1}{2} \delta+s+\tau_{j}, \delta\right) G\left(\frac{1}{2} \delta-s-\tau_{j}, \delta\right) G\left(1+s+\mu_{k}, \delta\right)} \\
& \left.\times \frac{G\left(\delta-s-\mu_{k}, \delta\right) G\left(\frac{1}{2} \delta+s+\nu_{l}, \delta\right) G\left(\frac{1}{2} \delta-s-\nu_{l}, \delta\right)}{G\left(1+\delta-s-\mu_{k}, \delta\right) G\left(1+\frac{1}{2} \delta+s+\nu_{l}, \delta\right) G\left(1+\frac{1}{2} \delta-s-\nu_{l}, \delta\right)}\right\} .
\end{aligned}
$$


The function $f(s)$ is determined by selecting terms in ' $s$ ' as opposed to ' $s+1$ '. Thus,

$$
f(s)=\pi^{\gamma} \prod_{i, j . k, l}\left\{\frac{G\left(1+\delta-s-\beta_{i}, \delta\right) G\left(1+\frac{1}{2} \delta-s-\tau_{j}, \delta\right) G\left(s+\mu_{k}, \delta\right) G\left(\frac{1}{2} \delta+s+\nu_{l}, \delta\right)}{G\left(s+\beta_{i}, \delta\right) G\left(\frac{1}{2} \delta+s+\tau_{j}, \delta\right) G\left(1+\delta-s-\mu_{k}, \delta\right) G\left(1+\frac{1}{2} \delta-s-\nu_{l}, \delta\right)}\right\} .
$$

It should be noted that this solution can be multiplied by any periodic function of period unity. The form of such a function is usually determined via information from the boundary value problem. An example of selection of the periodic function is given in $\S 5$ of this paper.

\section{Simplification of the general solution}

There are two practical methods by which the general solution (3.6) can be reduced to a tractable form. The first, and probably most useful, approach is simply to write each of the Barnes double gamma functions in its integral form using (2.14). The resulting integral expression will be valid, for all $\delta>0$, in a vertical strip of the complex $s$-plane. The second approach is to simplify (3.6) using the two independent recurrence relations for the Barnes double gamma function ((A 9) and (A 10)). If $I+J=K+L$ all the double gamma functions can be eliminated leaving finite products of gamma functions which can then be manipulated to obtain a solution in terms of standard trigonometrical functions. However, this approach usually provides a solution for rational values of $\delta$ only. This is because the exact solution to the fde is expressed in terms of Barnes' double gamma functions in which some fraction of $\delta$ enters the argument as well as being the parameter. Hence, in order to exploit recurrence relations (A 9) and (A 10), which relate $G(n+z, \delta)$ and $G(n \delta+z, \delta)$ to $G(z, \delta)$, it is necessary to insist that $\delta=2 q / p$ where $q, p$ are mutually prime and $p$ is odd. For the special case in which $I=J, K=L$ and $\alpha \beta_{i}, \alpha \tau_{j}, \alpha \mu_{k}, \alpha \nu_{l}$ are integers the fde may be solved exactly for all values of $\alpha$. The solution can usually be found by inspection: there is no need to employ either integral transformations or any sophisticated special functions. Finally, if $I+J \neq K+L$ then some simplification can be made using the recurrence relations, however, it will not be possible to eliminate all the Barnes' double gamma functions. In this case, it is best to utilize the integral form to simplify the solution.

The first approach is demonstrated here with an equation that was discussed by Van Lennep (1973) as being fundamental to a class of wedge diffraction problems. The fde is

$$
f(v+2 \alpha)-\cot \left\{\frac{1}{2}\left(v+\frac{1}{2} \pi\right)\right\} f(v-2 \alpha)=0 .
$$

As mentioned in $\S 1$, this, together with certain normalization conditions, defines Maliuzhnets' function. On making the substitutions $v=4 \alpha\left(s+\frac{1}{2}\right), g(s)=f(4 \alpha s)$ and $\delta=\pi / 2 \alpha$, this becomes

$$
\frac{g(s)}{g(s+1)}=\tan \left\{\frac{\pi}{\delta}\left(s+\frac{1}{2}+\frac{1}{4} \delta\right)\right\} .
$$

The quantities sine and cosine can be written in terms of the Barnes double gamma function using expressions (A 16) and (A 17). Thus, (4.2) can be written as

$$
\frac{g(s)}{g(s+1)}=\frac{G\left(s+\frac{1}{2}+\frac{1}{4} \delta, \delta\right) G\left(\frac{3}{4} \delta-s-\frac{1}{2}, \delta\right) G\left(\frac{1}{2}+\frac{1}{4} \delta-s, \delta\right) G\left(s+\frac{3}{2}+\frac{3}{4} \delta, \delta\right)}{G\left(s+\frac{3}{2}+\frac{1}{4} \delta, \delta\right) G\left(\frac{3}{4} \delta-s+\frac{1}{2}, \delta\right) G\left(\frac{1}{4} \delta-s-\frac{1}{2}, \delta\right) G\left(\frac{3}{4} \delta+\frac{1}{2}+s, \delta\right)} .
$$

It is a simple procedure to select terms in ' $s$ ' as opposed to ' $s+1$ ', and it follows that

$$
g(s)=\frac{G\left(s+\frac{1}{2}+\frac{1}{4} \delta, \delta\right) G\left(\frac{1}{2}+\frac{1}{4} \delta-s, \delta\right)}{G\left(\frac{1}{2}+\frac{3}{4} \delta-s, \delta\right) G\left(s+\frac{1}{2}+\frac{3}{4} \delta, \delta\right)}
$$


Now, each of the Barnes double gamma functions can be expressed as the exponential of an integral using (2.14). On combining these terms it is found that

$$
g(s)=\exp \left\{\int_{0}^{1} \frac{r^{s+\frac{1}{4} \delta-\frac{1}{2}}+r^{\frac{1}{4} \delta-s-\frac{1}{2}}}{(1-r)\left(r^{\frac{1}{2} \delta}+1\right) \ln r}+\frac{\delta r^{\delta-1}}{2 \ln r}-\delta\left(\frac{r^{\delta}-2}{r^{\delta}-1}\right) \frac{r^{\delta-1}}{\ln r} \mathrm{~d} r\right\} .
$$

The above integral is valid for $\delta>0$ and $-\frac{1}{2}-\frac{1}{4} \delta<\operatorname{Re}(s)<\frac{1}{2}+\frac{1}{4} \delta$. For values of $s$ outside this range $g(s)$ is determined using (4.2). The original function $f(v)$ is obtained by back substitution for $v$ and $\alpha$, i.e.

$$
f(v)=\exp \left\{\int_{0}^{1} \frac{r^{v+2 \alpha+\frac{1}{2} \pi-1}+r^{\frac{1}{2} \pi+2 \alpha-v-1}}{\left(1-r^{4 \alpha}\right)\left(r^{\pi}+1\right) \ln r}+\frac{\pi r^{2 \pi-1}}{4 \alpha \ln r}-\left(\frac{r^{2 \pi}-2}{r^{2 n}-1}\right) \frac{\pi r^{2 \pi-1}}{2 \alpha \ln r} \mathrm{~d} r\right\} .
$$

This is one solution to (4.1); further solutions may be obtained on multiplying by any function of period $4 \alpha$. Note that, Van Lennep obtains the following solution to the fde:

$$
f(v)=\prod_{n=1}^{\infty} \prod_{m=1}^{\infty}\left\{1-\left[\frac{v}{(2 n-1) \frac{1}{2} \pi+2 \alpha(2 m-1)}\right]^{2}\right\}^{(-1)^{n+1}} .
$$

This is the infinite product representation of the Maliuzhnets function (see Maliuzhnets, 1958) and thus differs from (4.6) by a multiplicative periodic factor or simply a constant. It follows that the Maliuzhnets function can be expressed as a combination of Barnes double gamma functions and this relationship is clarified in the Appendix.

The general solution (3.6) can also be simplified using the recurrence relations (A 9) and (A 10). This is the approach that was employed by Lawrie (1990), and it is demonstrated here using an fde discussed by Williams (1959). The equation is

$$
\sin \left\{\frac{1}{2}\left(w-\frac{3}{2} \pi\right)\right\} F(w)-\sin \left\{\frac{1}{2}\left(w-\frac{1}{2} \pi\right)\right\} F(w+2 \alpha)=0 .
$$

Williams shows that the solution can be written down in terms of the Barnes double gamma function. For the special cases $\alpha=\pi, 2 \pi$ he shows that this can be simplified in terms of Alexeiewsky's $G$ function and integral representations obtained. He also quotes a solution that is valid for $\alpha$ of the form $\alpha=p \pi / 2 q$ (where $p, q$ are relatively prime and $p$ is odd). However, this expression is not obtained through simplification of the general solution, instead, it is attributed to Lewy (1946) and Brillouet (1957). Here it is shown how the solution to (4.8), valid for $\alpha=p \pi / 2 q$, can be derived directly using the Barnes double gamma function and its recurrence relations.

On putting $w=2 \alpha s$ and $F(2 \alpha s)=f(s),(4.8)$ becomes

$$
\sin \left\{\alpha\left(s-\frac{3 \pi}{4 \alpha}\right)\right\} f(s)-\sin \left\{\alpha\left(s-\frac{\pi}{4 \alpha}\right)\right\} f(s+1)=0
$$

with solution

$$
f(s)=\frac{G\left(1+\delta-s+\frac{3}{4} \delta, \delta\right) G\left(s-\frac{1}{4} \delta, \delta\right)}{G\left(s-\frac{3}{4} \delta, \delta\right) G\left(1+\delta-s+\frac{1}{4} \delta, \delta\right)}
$$

where $\delta=\pi / \alpha$. For $\alpha=p \pi / 2 q$ this can be rewritten using the substitutions $x=$ $s-3 q / 2 p-2 q r / p$ and $y=1-s+q / 2 p-2 q(r-1) / p$ where $r=(p-1) / 2$. Then

$$
f(s)=\frac{G(y+q, 2 q / p) G(x+q, 2 q / p)}{G(x+2 q r / p, 2 q / p) G(y+2 q r / p, 2 q / p)} .
$$


The solution is now in a form that can be simplified using the recurrence relations, (A 9) and (A 10), for the Barnes double gamma function. It is found that

$$
f(s)=(2 \pi)^{r(1-2 q / p)}\left(\frac{2 q}{p}\right)^{-r q} \frac{\Pi_{m=0}^{q-1} \Gamma\{p(m+x) / 2 q\} \Gamma\{p(m+y) / 2 q\}}{\prod_{m-0}^{r-1} \Gamma(x+2 q m / p) \Gamma(y+2 q m / p)} .
$$

To simplify this further it is necessary to rewrite $x$ and $y$ in terms of $p, q$ and $s$. Then, on replacing $(m+1-q)$ with $-m$ in the second gamma function of the numerator and $(m-r+1)$ with $-m$ in the first gamma function of the denominator, it is found that

$$
f(s)=(2 \pi)^{r(1-2 q / p)}\left(\frac{2 q}{p}\right)^{-r q} \frac{\prod_{m-0}^{q-1} \Gamma\left\{p(m+s) / 2 q-\frac{3}{4}-r\right\} \Gamma\left\{1-p(m+s) / 2 q+\frac{3}{4}\right\}}{\prod_{m-1}^{r} \Gamma\{s-3 q / 2 p-2 q m / p\} \Gamma\{1-s-3 q / 2 p+2 q m / p-q\}} .
$$

This is easily rearranged to obtain

$$
f(s)=(2 \pi)^{r(1-2 q / p)}(-1)^{r q} \pi^{q-r} \frac{\prod_{m-1}^{r} \sin \{\pi(s-3 q / 2 p-2 q m / p)\}}{\prod_{m=0}^{q-1} \sin \{p \pi(m+s) / 2 q-3 \pi / 4\}} .
$$

It follows that the solution to $(4.8)$ is

$$
F(w)=(2 \pi)^{r(1-2 q / p)}(-1)^{r q} \pi^{q-r} \frac{\prod_{m-1}^{r} \sin \{q(w-3 \pi / 2-2 \pi m) / p\}}{\prod_{m-0}^{q-1} \sin \left\{\frac{1}{2} w+p \pi m / 2 q-\frac{3}{4} \pi\right\}} .
$$

Expression (4.15) is a valid solution to (4.8) for $\alpha$ of the form $\alpha=p \pi / 2 q$. It should be noted that the solution quoted by Williams (1959) appears to have a factor of 2 missing in the denominator.

Provided $I+J=K+L$ (see (3.3) and (3.4)) this approach can be used to simplify the general solution for rational values of $\delta$. Despite the restrictions on $\delta$ (and thus on $\alpha$ ), the resulting expressions are useful since they are composed of finite products of elementary trigonometrical functions.

\section{Applications of results to a mixed boundary value problem in a wedge}

As a final illustration of the utility of the results of $\S 2$, we consider the problem of determining the motion of the contact point between fluid/solid and air at the tip of a wedge of fluid which has just snapped off from another body of fluid. The initial configuration is a semi-infinite wedge with apex angle $\alpha$. To model the snapping process, it is assumed that the fluid moves instantaneously to form the contact angle $\beta$. The angle between the solid and the free surface remains fixed for subsequent time whilst the original wedge structure is retained in the far-field. The first study of this was carried out by Keller \& Miksis (1983) who reformulated the fluid mechanics problem as a nonlinear integrodifferential equation. This equation was solved numerically and some interesting results presented. That a bounded solution exists in the neighbourhood of the contact line was first pointed out by Tayler (1972). A linearized version of the far-field problem was carried out by Lawrie (1990) for wedges of arbitrary angle. The far-field solution fails close to the contact point and in this region the free surface was modelled by a circular arc. A nonlinear theory, valid for slender wedges was developed by King (1991), and resulted in a novel 


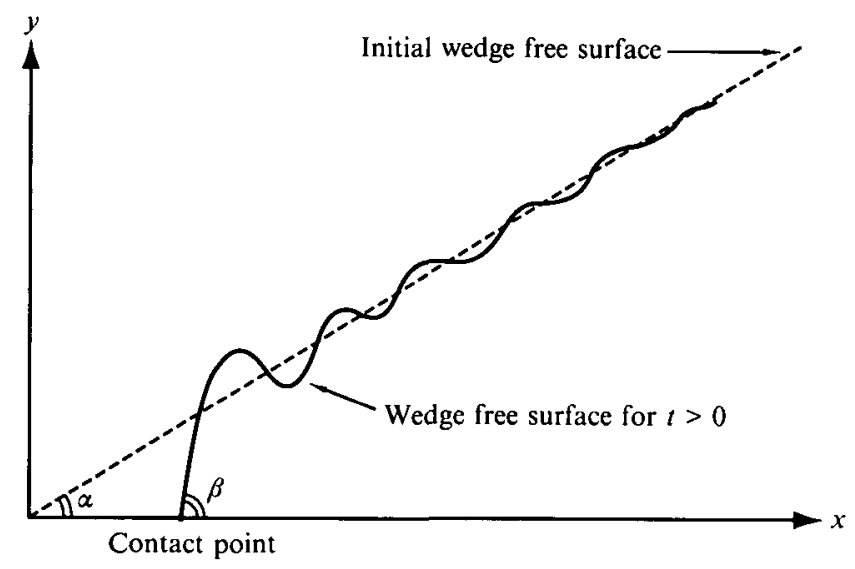

Figure 1.

eigenvalue problem whose solution was used to determine the free surface of the wedge and the motion of the contact point.

The linear theory for such flows is now reconsidered and the region close to the contact point is examined using a formal perturbation theory. The flow is surface tension driven and the geometry has no natural length scale; thus, following Keller \& Miksis (1983), the boundary value problem may be written in similarity form. Using similarity variables $\zeta=x\left(\rho / \sigma t^{2}\right)^{\frac{1}{3}}, \eta=y\left(\rho / \sigma t^{2}\right)^{\frac{1}{3}}$, where $(x, y)$ are standard Cartesian coordinates with origin at the undisturbed wedge apex (see Figure 1), the dimensionless velocity potential $\phi$, and free surface $Y_{s}$, satisfy

$$
\phi_{\xi \xi}+\phi_{m}=0, \quad \xi, \eta \in D_{\alpha, \beta},
$$

where

$$
D_{\alpha, \beta}=\left\{(\xi, \eta): \xi_{c}<\xi<\infty, 0<\eta<Y_{s}(\xi)\right\},
$$

subject to the kinematic and Bernoulli conditions at the free surface, which take the form

$$
\phi_{\eta}=-\frac{2}{3} \xi Y_{s}^{\prime}+\frac{2}{3} Y_{s}+\phi_{\xi} Y_{s}^{\prime}
$$

and

$$
\frac{1}{3} \phi-\frac{2}{3}\left\{\xi \phi_{\xi}+\eta \phi_{\eta}\right\}+\frac{1}{2}\left\{\phi_{\xi}^{2}+\phi_{\eta}^{2}\right\}+\frac{Y_{s}^{\prime \prime}}{\left\{1+\left(Y_{s}^{\prime}\right)^{2}\right\}^{\frac{3}{2}}}=0 .
$$

At the solid surface the no-slip condition is applied, that is $\phi_{\eta}=0$ on $\eta=0$. The conditions at the contact point are $Y_{s}\left(\xi_{c}\right)=0, Y_{s}^{\prime}\left(\xi_{c}\right)=\tan \beta$ and, to ensure a bounded solution, it is assumed that $\phi \rightarrow 0$ as $\xi \rightarrow \infty$, and $Y_{s}(\xi) \sim \xi \tan \alpha$ as $\xi \rightarrow \infty$.

Under the assumption that $\beta=\alpha+\epsilon, \epsilon \ll 1$, a formal linearization is obtained by writing

$$
\begin{gathered}
\phi=\epsilon \tilde{\phi}+\ldots, \\
Y_{s}=\xi \tan \alpha+\epsilon \tilde{Y}+\ldots, \\
\xi_{c}=\epsilon \tilde{\xi}_{c}+\ldots,
\end{gathered}
$$

with a corresponding domain decomposition in the form

$$
D_{\alpha, \beta}=D_{\alpha, \alpha} \cup D_{\alpha, \epsilon} \cup \ldots,
$$

where

$$
D_{\alpha \alpha}=\{(\xi, \eta): 0<\xi<\infty, 0<\eta<\xi \tan \alpha\} \text {. }
$$


Here, the position of the contact point is given, to leading order, by $\tilde{\xi}_{c}$. On substituting expansions (5.1)-(5.3) into the full nonlinear boundary value problem, the leading order linear problem is found to be

$$
\tilde{\phi}_{x x}+\tilde{\phi}_{\eta \eta}=0, \quad \xi, \eta \in D_{\alpha, \alpha}
$$

subject to the boundary conditions

$$
\begin{gathered}
\frac{1}{3} \tilde{\phi}-\frac{2}{3} \xi\left\{\tilde{\phi}_{\xi}+\tilde{\phi}_{\eta} \tan \alpha\right\}+\tilde{Y}^{\prime \prime}(\xi) \cos ^{3} \alpha=0, \quad \eta=\xi \tan \alpha, \\
\tilde{\phi}_{\eta}=-\frac{2}{3} \xi \tilde{Y}^{\prime}+\frac{2}{3} \tilde{Y}+\tilde{\phi}_{\xi} \tan \alpha, \quad \eta=\xi \tan \alpha, \\
\tilde{\phi}_{\eta}=0, \quad \eta=0,
\end{gathered}
$$

and with contact conditions given by

$$
\begin{gathered}
\tilde{\xi}_{c}=-\tilde{Y}(0) \cot \alpha, \\
T^{\prime}(0)=\sec ^{2} \alpha .
\end{gathered}
$$

As before, it is assumed that $\tilde{Y}(\xi), \tilde{\phi} \rightarrow 0$ as $\xi \rightarrow \infty$.

It is expedient to write this boundary value problem in terms of polar coordinates, $\xi=r \cos \theta, \eta=r \sin \theta, \zeta=\tilde{Y} \cos \alpha$. It then takes the form

$$
\tilde{\phi}_{r r}+\frac{1}{r} \tilde{\phi}_{r}+\frac{1}{r^{2}} \tilde{\phi}_{\theta 0}=0
$$

subject to

$$
\begin{gathered}
\frac{1}{r} \tilde{\phi}_{\theta}-\frac{2}{3} r \zeta^{\prime}+\frac{2}{3} \zeta=0, \quad \theta=\alpha \\
\frac{1}{3} \tilde{\phi}-\frac{2}{3} r \tilde{\phi}_{r}+\zeta^{\prime \prime}=0, \quad \theta=\alpha \\
\tilde{\phi}_{\theta}=0, \quad \theta=0 \\
\zeta(0)=-\tilde{\xi}_{c} \sin \alpha, \\
\zeta^{\prime}(0)=1,
\end{gathered}
$$

together with the usual constraints that $\tilde{\phi}, \tilde{\zeta} \rightarrow \infty$ as $r \rightarrow \infty$. This mixed boundary value problem, excluding conditions (5.4)-(5.5), was solved by Lawrie (1990) as part of the farfield solution to the linear problem; we quote the solution as

$$
\begin{gathered}
\tilde{\phi}(r, \theta)=\frac{1}{2 \pi i} \int_{c-i \infty}^{c+i \infty} \Gamma(s) \Gamma\left(\frac{-2 s}{3}-\frac{1}{3}\right) f(s) \cos (\theta s) r^{-5} \mathrm{~d} s, \\
\zeta(r)=-\frac{1}{2 \pi i} \int_{c-i \infty}^{c+i \infty} \Gamma(s) \Gamma\left(\frac{-2 s}{3}-\frac{2}{3}\right) f(s-1) \sin (\alpha(1-s)) r^{-s} \mathrm{~d} s
\end{gathered}
$$

where $0<c<c_{0}$ is chosen so that the integrals converge and give rise to a bounded solution, and $f(s)$ satisfies the fde

$$
f(s) \cos (\alpha s)+f(s-3) \sin \{\alpha(s-3)\}=0, \quad 0<s<c_{0} .
$$


An examination of the integrand in (5.7) shows that a double pole exists at $s=-1$. This gives rise to a $\ln (r)$, thus, to ensure that $\zeta(r)$ is bounded the condition

$$
f(-2)=0
$$

is imposed. Similarly, in the integral expression for $\phi(r, \theta)$ there is a pole at $s=-\frac{1}{2}$ which results in the velocity being $0\left(r^{-\frac{1}{2}}\right)$ as $r \rightarrow 0$. Again, this unbounded behaviour is eliminated by choosing

$$
f\left(-\frac{1}{2}\right)=0 .
$$

The form of $\zeta(r)$ as $r \rightarrow 0$ is determined by closing the Mellin inversion integral contour to the left of the origin and using the calculus of residues. It is found that

$$
\begin{gathered}
\zeta(0)=\Gamma\left(\frac{-2 s}{3}\right) f(-1) \sin \alpha, \\
\zeta^{\prime}(0)=-\sin 2 \alpha \lim _{s \rightarrow-1}\left\{\Gamma\left(\frac{-2 s}{3}-\frac{2}{3}\right) f(s-1)\right\} .
\end{gathered}
$$

Equations (5.5) and (5.12) can be used to give a third condition on the solution of the difference equation (5.8), i.e.

$$
\lim _{s \rightarrow-1}\left\{\Gamma\left(\frac{-2 s}{3}-\frac{2}{3}\right) f(s-1)\right\}=-\csc 2 \alpha
$$

whilst (5.4) and (5.11) allow the position of the contact point to be expressed as

$$
\tilde{\xi}_{c}=-\Gamma\left(\frac{-2}{3}\right) f(-1)=\frac{\Gamma(-2 / 3) \cos (2 \alpha) f(2)}{\sin \alpha} .
$$

The solution to the boundary value problem is now complete apart from the specification of the solution to the third order difference equation (5.8). This is firstly reduced to a first order equation using standard methods and then a solution can be found in terms of the double gamma function in the form

$$
f(s)=C(s) \frac{G\left(\frac{1}{2} \delta+\frac{1}{3} s+1, \delta\right) G\left(1-\frac{1}{3} s, \delta\right)}{G\left(\frac{1}{2} \delta-\frac{1}{3} s, \delta\right) G\left(\frac{1}{3} s+\delta, \delta\right)},
$$

where $\delta=\pi / 3 \alpha$ and $C(s)$ is a function of period 3. Application of conditions (5.9) and (5.10) leads to $C(s)=C_{0}(\alpha) \sin \frac{1}{3} \pi(2 s+1)$; the remaining condition, (5.13), gives

$$
C_{0}(\alpha)=-\frac{1}{\pi \cos \alpha} \frac{G\left(\frac{1}{2} \delta-\frac{1}{3}, \delta\right) G\left(\frac{1}{3}+\delta, \delta\right)}{G\left(\frac{1}{2} \delta+\frac{4}{3}, \delta\right) G\left(\frac{2}{3}, \delta\right)}
$$

which allows, after a little algebra, the position of the contact point $\tilde{\xi}_{c}$ to be written as

$$
\tilde{\xi}_{c}=\frac{3 \sqrt{3} \delta^{\frac{1}{3}} \cos \alpha}{2 \pi \Gamma\left(\frac{2}{3}\right) \sin 2 \alpha}\left\{\frac{\Gamma\left(\frac{1}{3}\right) \Gamma(2 / 3 \delta) G\left(\frac{1}{2} \delta+\frac{2}{3}, \delta\right) G\left(\frac{4}{3}, \delta\right)}{\Gamma(1 / 3 \delta) G\left(\frac{1}{2} \delta+\frac{1}{3}\right) G\left(\frac{5}{3}+\delta\right)}\right\}^{2} .
$$

This expression is easily computed using a simple subroutine call to evaluate the double gamma function by a numerical quadrature of the integral representation of $\S 2$. Some results are shown in Figure 2. 


\section{Discussion and conclusion}

The unsymmetric double gamma function as defined by Barnes (1899) is one of the lesserknown special functions of applied mathematics. Nevertheless, it has some interesting properties. In this article its relationship to the well-known gamma function and the standard trignonmetric functions has been exploited to provide an algorithm by which a class of functional difference equations with trigonometrical coefficients can be solved by inspection. Further properties, namely the recurrence relations (A 9) and (A 10), or the integral representation derived in $\$ 2$, enable the solution to be simplified further. The practical application of this approach has been demonstrated in $\S 5$, where the contact line displacement of a surface tension-driven fluid wedge is determined as a quotient of the Barnes double gamma functions. A single integral expression for the displacement is obtained via the integral form (2.14). This is then evaluated and plotted against $\alpha$, the initial wedge angle (see Figure 2).

Of course, the Barnes double gamma function is not the only means by which the first order fdes with trigonometric coefficients can be solved. The Maliuzhnets function was initially derived (Maliuzhnets, 1958) as the solution to (1.2), together with specified normalization and radiation conditions. It has subsequently been used to solve further fdes of the type considered in this paper. However, considering the form of (1.2), it is no surprise that the Maliuzhnets function can be expressed as a quotient of Barnes double gamma functions (see Appendix). A simple change of argument enables (1.2) to be written as

$$
\frac{g(s)}{g(s+1)}=\tan \left\{\pi\left(s+\frac{1}{2}+\frac{1}{4} \delta\right) / \delta\right\},
$$

which can be expressed as

$$
\frac{g_{1}(s) g_{2}(s) g_{3}(s) g_{4}(s)}{g_{1}(s+1) g_{2}(s+1) g_{3}(s+1) g_{4}(s+1)}=\frac{\Gamma\left\{\left(3 \delta / 4+s+\frac{1}{2}\right) / \delta\right\} \Gamma\left\{\left(\delta / 4-s-\frac{1}{2}\right) / \delta\right\}}{\Gamma\left\{\left(\delta / 4+s+\frac{1}{2}\right) / \delta\right\} \Gamma\left\{\left(3 \delta / 4-s-\frac{1}{2}\right) / \delta\right\}}
$$

where $g(s)=g_{1}(s) g_{2}(s) g_{3}(s) g_{4}(s)$. By comparison, the Barnes double gamma function is defined by $(2.1)$, i.e.

$$
\frac{G(z, \delta)}{G(z+1, \delta)}=\frac{1}{\Gamma(z / \delta)}
$$

together with the normalization condition $G(1, \delta)=1$. Linear fdes are multiplicative and thus it is clear that, with correct choice of $z$ and neglecting any arbitrary periodic functions, $g_{j}(s), j=1,2,3,4$ is simply some constant times either a Barnes double gamma function or its reciprocal. It must therefore be concluded that, explicitly or implicitly, the Barnes double gamma function is fundamental to the solution of any first order fde with trigonometric coefficients.

\section{Appendix The Barnes double gamma function}

The unsymmetric double gamma function as defined by Barnes (1899) is the solution to the difference equation

$$
G(z+1, \delta)=\Gamma(z / \delta) G(z, \delta)
$$

with condition

$$
G(1, \delta)=1 .
$$




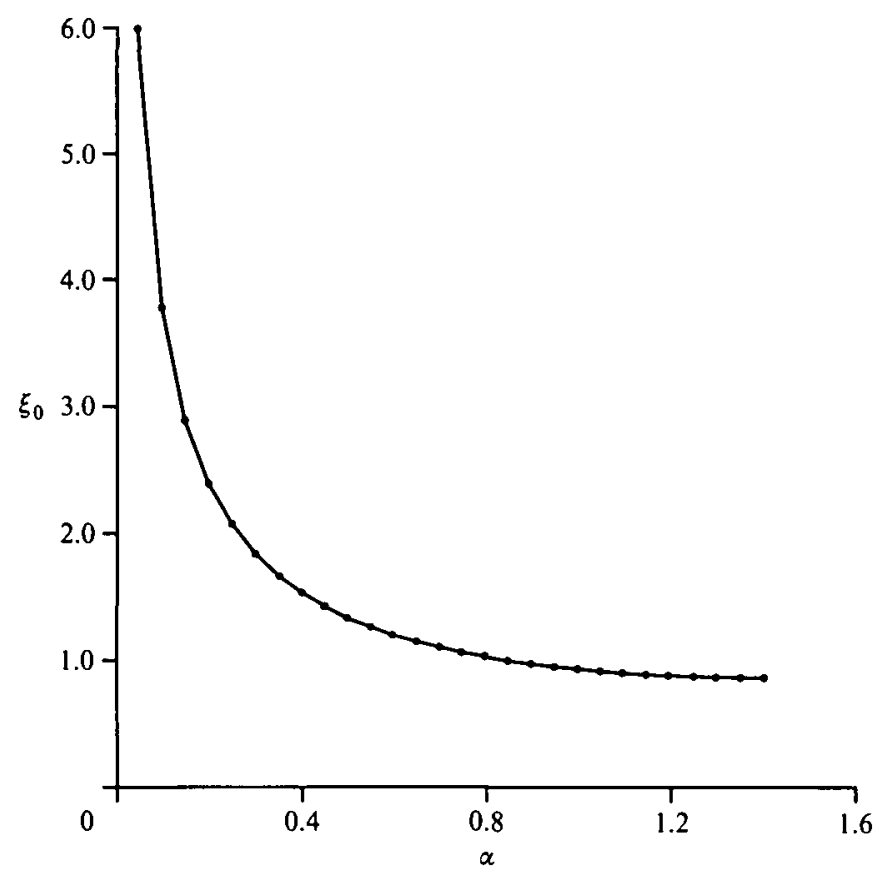

FIGURE 2.

Barnes shows that the function $G(z, \delta)$ can be expressed as an infinite product of gamma functions with argument differing by multiples of $\delta$. In fact,

$$
G(z, \delta)=\frac{\mathrm{e}^{A(\delta) z+B(\delta) z^{2} / 2}}{\delta \Gamma(z)} \prod_{m=1}^{\infty} \frac{\Gamma(m \delta)}{\Gamma(z+m \delta)} \mathrm{e}^{z \psi^{\prime}(m \delta)+z^{2} \psi^{\prime}(m \delta) / 2},
$$

where $\psi(\cdot)$ denotes the Psi or Digamma function defined by

$$
\psi(z)=\frac{\mathrm{d}}{\mathrm{d} z}\{\ln \Gamma z\}=\frac{\Gamma^{\prime}(z)}{\Gamma(z)} .
$$

Note that, $\psi(1)=-\gamma$ where $\gamma=0.577215 \ldots$ is Euler's constant. The quantities $A(\delta)$ and $B(\delta)$ are here defined by

$$
A(\delta)=\frac{\gamma}{2 \delta}+\ln (\delta)-\sum_{m=1}^{\infty}\left\{\psi(m \delta)-\ln (m \delta)+\frac{1}{2 m \delta}\right\}
$$

and

$$
B(\delta)=-\frac{\gamma}{\delta}-\sum_{m=1}^{\infty}\left\{\psi^{\prime}(m \delta)-\frac{1}{m \delta}\right\}
$$

where the prime indicates differentiation with respect to the argument. Each of the above sums converge at rate $1 / n^{2}$ so that $A(\delta)$ and $B(\delta)$ are functions of $\delta$ only. It should be noted that Barnes expresses these functions in a slightly different, though equivalent, form. By inspection of (A 3), it is clear that the Barnes double gamma function has no poles but an infinite number of zeros given by

$$
z=-(m \delta+n), \quad m=0,1,2, \ldots, n=0,1,2, \ldots
$$


The Barnes double gamma function also satisfies the difference equation

$$
G(z+\delta, \delta)=(2 \pi)^{(\delta-1) / 2} \delta^{-z+\frac{1}{2}} \Gamma(z) G(z, \delta) .
$$

Using (A 3) and (A 8) two recurrence relations can be deduced, these are

$$
G(n+z, \delta)=G(z, \delta) \prod_{m=0}^{n-1} \Gamma\{(m+z) / \delta\}
$$

and

$$
G(z+n \delta, \delta)=(2 \pi)^{n(\delta-1) / 2} \delta^{-n z-n(n-1) \delta / 2+n / 2} G(z, \delta) \prod_{m=0}^{n-1} \Gamma\{z+m \delta\} .
$$

It is possible to derive the integral representation, obtained in $\S 2$, for the Barnes double directly from (A 3). The method involves first taking logarithms of both sides of the equation and expressing the terms of each summation in integral form. Thus,

$$
\begin{aligned}
& \ln G(z, \delta)=(z-1) \ln \delta+z(1-z) \gamma /(2 \delta)-\ln \Gamma(z) \\
& -z \sum_{m=1}^{\infty} \frac{2}{\delta^{2}} \int_{0}^{\infty} \frac{t}{\left(\mathrm{e}^{2 \pi t}-1\right)\left(m^{2}+t^{2} / \delta^{2}\right)} \mathrm{d} t \\
& +\frac{z^{2}}{2} \sum_{m-1}^{\infty} \int_{0}^{1}\left\{\frac{1}{r}+\frac{\ln r}{r(1-r)}\right\}\left(r^{\delta}\right)^{m} \mathrm{~d} r \\
& -\sum_{m=1}^{\infty} \int_{0}^{1}\left\{\frac{1-r^{z}}{\ln r}+z+\frac{z^{2}}{2} \ln r\right\} \frac{\left(r^{\delta}\right)^{m}}{r(1-r)} \mathrm{d} r .
\end{aligned}
$$

The orders of summation and integration may now be interchanged and, on performing, the summations it is found that

$$
\begin{aligned}
\ln G(z, \delta)= & (z-1) \ln \delta+z(1-z) \gamma /(2 \delta)-\ln \Gamma(z) \\
& -\frac{z \pi}{\delta} \int_{0}^{\infty}\left\{\frac{1+\mathrm{e}^{-2 \pi t / \delta}}{1-\mathrm{e}^{-2 \pi t / \delta}}-\frac{\delta}{\pi t}\right\} \frac{\mathrm{d} t}{\left(\mathrm{e}^{2 \pi t}-1\right)} \\
& -\frac{z^{2}}{2} \int_{0}^{1}\left\{1+\frac{\ln r}{1-r}\right\} \frac{r^{\delta-1}}{\left(r^{\delta}-1\right)} \mathrm{d} r \\
& -\int_{0}^{1}\left\{\frac{r^{z}-1}{\ln r}-z-\frac{z^{2}}{2} \ln r\right\} \frac{r^{\delta-1}}{(1-r)\left(r^{\delta}-1\right)} \mathrm{d} r .
\end{aligned}
$$

On making the substitution $r=\mathrm{e}^{-2 \pi t / \delta}$ in the first integral, expressing the quantities $\gamma, \ln \delta$ and $\ln \Gamma(z)$ in integral form and rearranging it is found that

$$
\begin{aligned}
G(z, \delta)=\exp \left\{\int _ { 0 } ^ { 1 } \left\{\frac{r^{z-1}}{(r-1)\left(r^{\delta}-1\right)}-\frac{z^{2} r^{\delta-1}}{2 \delta}+z r^{\delta-1}\left\{\frac{r^{\delta}-2}{r^{\delta}-1}+\frac{1}{2 \delta}\right\}\right.\right. \\
\left.\left.-r^{\delta-1}+\frac{1}{(r-1)}-\frac{r^{\delta-1}}{(r-1)\left(r^{\delta}-1\right)}\right\} \frac{\mathrm{d} r}{\ln r}\right\},
\end{aligned}
$$

which is in agreement with the result of $\S 2$. The above integral representation can be 


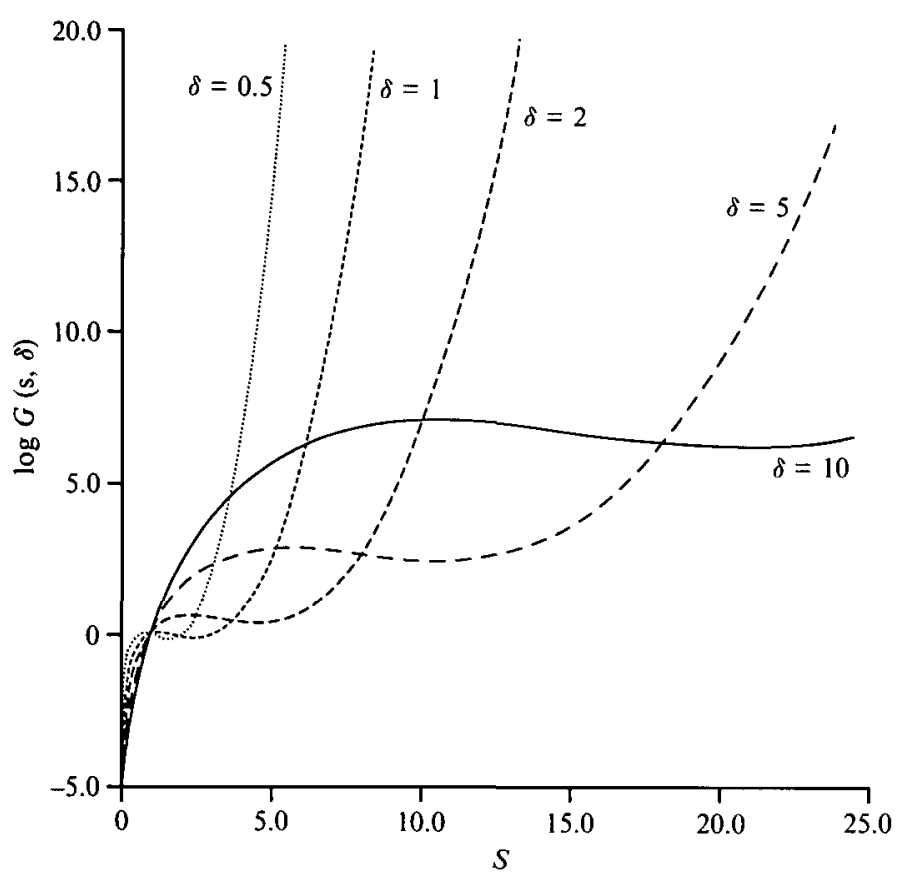

FIGURE 3.

employed to obtain the duplication formula for the Barnes double gamma function. It is found that

$$
G(2 z, \delta)=\frac{G(z, \delta / 2) G(z+1 / 2, \delta / 2)}{G\left(\frac{1}{2}, \delta / 2\right)} .
$$

Higher order multiplication formulae can also be obtained. It is a simple matter to compute the Barnes doubles gamma function using the integral representation (A 13). Figure 3 shows $\ln G(z, \delta)$ against $z>0$ for various $\delta$; note that each of the curves tends to $-\infty$ as $z \rightarrow 0$, is zero when $z=1$ and then grows exponentially as $z \rightarrow \infty$.

The integral expression (A 13) is a finite integral of the Mellin transform type. However, it is worthwhile pointing out that alternative forms do exist and may occasionally be of more use. For example, by making the substitution $r=\mathrm{e}^{-x}$ in (A 13), it is found that

$$
G(s, \delta)=\exp \left\{\int_{0}^{\infty}\left\{\frac{1-\mathrm{e}^{-s x}}{\left(\mathrm{e}^{-x}-1\right)\left(\mathrm{e}^{-\delta x}-1\right)}+\frac{s \mathrm{e}^{-\delta x}}{\left(\mathrm{e}^{-\delta x}-1\right)}+(s-1)\left(\frac{s}{2 \delta}-1\right) \mathrm{e}^{-\delta x}\right\} \frac{\mathrm{d} x}{x}\right\} .
$$

It is useful to relate the Barnes double gamma function to other well known functions. Of particular relevance to this work are the trigonometric functions all of which can be expressed in terms of Barnes' function. For example, the function $\sin (\pi z / \delta)$ can be expressed in terms of Gamma functions as follows:

$$
\sin (\pi z / \delta)=\frac{\pi}{\Gamma(z / \delta) \Gamma(1-z / \delta)}
$$


On putting $n=1$ in (A 9), the gamma functions can be rewritten as a quotient of double gamma functions and it follows that

$$
\sin (\pi z / \delta)=\pi \frac{G(z, \delta) G(\delta-z, \delta)}{G(1+z, \delta) G(1+\delta-z, \delta)} .
$$

The equivalent expression for $\cos (\pi z / \delta)$ is

$$
\cos (\pi z / \delta)=\pi \frac{G(\delta / 2+z, \delta) G(\delta / 2-z, \delta)}{G(1+\delta / 2+z, \delta) G(1+\delta / 2-z, \delta)} .
$$

Alexeiewsky's $\mathscr{G}$ function is also related to the Barnes double gamma function, corresponding to the case $\delta=1$, i.e.

$$
G(z, 1)=\mathscr{G}(z)
$$

where $\mathscr{G}(z)$ satisfies

$$
\mathscr{G}(z+1)=\Gamma(z) \mathscr{G}(z), \quad \mathscr{G}(1)=1 .
$$

The reader is referred to Whittaker \& Watson (1927) for further details regarding Alexieiwsky's $\mathscr{G}$ function.

Finally, Maliuzhnets' function may be expressed in terms of the Barnes double gamma as follows:

$$
M_{\alpha}(v)=\left\{\frac{G\left(3 \delta / 4+\frac{1}{2}, \delta\right)}{G\left(\delta / 4+\frac{1}{2}, \delta\right)}\right\}^{2} \frac{G\left(\delta / 4+\frac{1}{2}+v \delta / 2 \pi, \delta\right) G\left(\delta / 4+\frac{1}{2}-v \delta / 2 \pi, \delta\right)}{G\left(3 \delta / 4+\frac{1}{2}+v \delta / 2 \pi, \delta\right) G\left(3 \delta / 4+\frac{1}{2}-v \delta / 2 \pi, \delta\right)} .
$$

Here $M_{\alpha}(v)$ satisfies (4.1) and $\delta=\pi / 2 \alpha$. On using the integral expression (A 13), and making the substitution $r=\mathrm{e}^{-x}$, where $r$ is the variable of integration, it may be confirmed that

$$
M_{\alpha}(v)=\exp \left\{-\frac{1}{2} \int_{0}^{\infty} \frac{\cosh (v x)-1}{x \cosh (x \pi / 2) \sinh (2 x \alpha)} \mathrm{d} x\right\}
$$

which agrees with the integral expression given by Lipszyc (1975). Further infinite product and integral representations for the Maliuzhnets function are given by Maliuzhnets (1958), Lipszyc (1975) and Hongo (1980).

\section{Acknowledgement}

The authors are grateful to the referee for several helpful suggestions which have greatly improved the presentation of this paper.

\section{References}

Abramowitz, M. \& Stegun, I. A. 1972 Handbook of Mathematical Functions. Dover.

Barnes, E. W. 1899 The genesis of the double gamma functions. Proc. Lond. Math. Soc. 31, 358-381.

Brillouet, G. 1957 Publ. Sci. Tech. Ministeair, Paris, no. 329.

Ehrenmark, U. T. 1987 Far field asymptotics of the two-dimensional linearised sloping beach problem. SIAM J. Appl. Math. 47 (5), 965-981. 
Evans, D. V. 1984 The solution of a class of boundary-value problems with smoothly varying boundary conditions. Q. J. Mech. Appl. Math. 34 (4), 521-536.

Faulkner, T. R. 1965 Diffraction by a perfectly conducting wedge in an anisotropic plasma. Proc. Camb. Phil. Soc. 61, 767-776.

HoNGo, K. 1980 Polynomial approximation of Maliuzhinets' function. IEEE Trans. Ant. Prop. 34, 942-947.

IsaCCSON, E. 1950 Water waves over a sloping bottom. Comm. Pure Appl. Math. 3, 11-31.

Keller, J. B. \& Miksis, M. J. 1983 Surface tension driven flows. SIAM J. Appl. Math. 43, 268-277.

KING, A. C. 1991 Moving contact lines in slender fluid wedges. Q. J. Appl. Math. 44 (2), 173-192.

Kolter, W. T. 1955 On the diffusion of load from a stiffner into a sheet. Q.J. Mech. Appl. Math. $8(2), 164-178$.

Lauwerier, H. A. 1961 Solutions of the equation of Helmholtz in an angle. IV. Proc. Ned. Akad. Werensch. A64 (23), 348-359.

LAWRIE, J. B. 1990 Surface tension driven flow in a wedge. Q. J. Mech. Appl. Math. 43 (2), 251-273.

Lewy, H. 1946 Water waves on sloping beaches. Bull. Amer. Math. Soc. 52, 737-775.

LiPSZYC, K. 1975 One-dimensional model of the rearrangement and dissociation processes and the Faddeev equations. II Phys. Rev. D11 (6), 1649-1661.

LiPSZYC, K. 1980 On the application of the Sommerfeld-Maluzhinetz transformation to some onedimensional three-particle problems. J. Math. Phys. 21 (5), 1092-1102.

MaliuzhNeTS, G. D. 1958 Excitation, reflection and emission of surface waves from a wedge with given face impedances. Sov. Phys. Dokl. 3, 752-755.

Milne-Thomson, L. M. 1933 The Calculus of Finite Differences. Macmillan.

NoBle, B. 1958 Methods Based on the Wiener-Hopf Technique. Pergamon.

PaCKHAM, B. A. 1989 A note on generalized edge waves on a sloping beach. Q.J. Mech. Appl. Math. $42(3), 441-446$.

PETERS, A. S. 1952 Water waves over sloping beaches and the solution of a mixed boundary value problem for $\Delta^{2} \phi-k^{2} \phi=0$ in a sector. Comm. Pure Appl. Math. 5, 87-108.

Roseau, M. 1958 Short waves parallel to the shore over a sloping beach. Comm. Pure Appl. Math. 11, 433-493.

RoSEAU, M. 1976 Asymptotic Wave Theory. North-Holland.

TAYler, A. B. 1972 Singularities at flow separation. Quart. J. Mech. Appl. Math. 26, 153-164.

TITChmaRSH, E. C. 1948 Introduction to the Theory of Fourier Integrals, 2nd edition. Oxford University Press.

Tuzhilin, A. A. 1971 The theory of Malyuzhinets functional equations IV. Differential 'nye Uraveneniya 7, 968-976. (Translation.)

Van DanTzIG, D. 1958 Solutions of the equation of Helmholtz in an angle with vanishing directional derivatives along each side. Kon. Ned. Akad. v. Wet. Proc. A61(4), 384-398.

VAN LenneP, A. G. R. 1973 The Kernel of Sommerfield's transform as solution to difference equations for a class of diffraction problems. J. Appl. Phys., 45, 4401-4405.

VARLeY, E. \& Walker, J. D. A. 1989 A method for solving Integrodifferential Equations. I.M.A. J. Appl. Maths. 43, 11-46.

Whittaker, E. T. \& Watson, G. N. 1927 A Course of Modern Analysis, 4th Edition Cambridge University Press.

Williams, W. E. 1959 Diffraction of an E-polarized plane wave by an imperfectly conducting wedge. Proc. $R$. Soc. Lond. A 252, 376-393. 